\title{
Simplified 2D Bidomain Model of Whole Heart Electrical Activity and ECG Generation
}

\author{
Siniša Sovilj ${ }^{1}$, Ratko Magjarević ${ }^{1}$, Amr Al Abed ${ }^{2}$, Nigel H. Lovell ${ }^{2}$, Socrates Dokos ${ }^{2}$ \\ ${ }^{1}$ Faculty of Electrical Engineering and Computing, University of Zagreb, 10000, Zagreb, Croatia, sinisa.sovilj@ieee.org \\ ${ }^{2}$ Graduate School of Biomedical Engineering, University of New South Wales, 2052, Sydney, Australia, s.dokos@unsw.edu.au
}

\begin{abstract}
The aim of this study was the development of a geometrically simple and highly computationally-efficient two dimensional (2D) biophysical model of whole heart electrical activity, incorporating spontaneous activation of the sinoatrial node (SAN), the specialized conduction system, and realistic surface ECG morphology computed on the torso. The FitzHugh-Nagumo (FHN) equations were incorporated into a bidomain finite element model of cardiac electrical activity, which was comprised of a simplified geometry of the whole heart with the blood cavities, the lungs and the torso as an extracellular volume conductor. To model the ECG, we placed four electrodes on the surface of the torso to simulate three Einthoven leads $V_{I}, V_{I I}$ and $V_{\text {III }}$ from the standard 12-lead system. The 2D model was able to reconstruct ECG morphology on the torso from action potentials generated at various regions of the heart, including the sinoatrial node, atria, atrioventricular node, His bundle, bundle branches, Purkinje fibers, and ventricles. Our 2D cardiac model offers a good compromise between computational load and model complexity, and can be used as a first step towards three dimensional (3D) ECG models with more complex, precise and accurate geometry of anatomical structures, to investigate the effect of various cardiac electrophysiological parameters on ECG morphology.
\end{abstract}

Keywords: Heart, bidomain model, ECG, cardiac activation.

\section{INTRODUCTION}

Cis OMPUTATIONAL cardiac electrophysiological modeling is a growing field in which cardiac biophysical models are becoming increasingly elaborate, complex and intricate, expanding our knowledge on electrical activation of the heart.

Biophysical models of cardiac electrical activity and resulting torso surface potentials are becoming increasingly detailed and computationally onerous: occasionally requiring even the use of supercomputers [1-4]. This is especially true for bidomain element models of cardiac electrical activity, which couple transmembrane cellular potentials to extracellular fields, requiring relatively high degrees of freedom to solve for all the dependent variables. We have developed a simplified computationally-efficient two-dimensional (2D) model of cardiac electrical activity that can generate spontaneous periodic activation within the sinoatrial node (SAN) and can reproduce a realistic surface electrocardiogram (ECG) on the torso. The model can be used as a basis for quick and reliable a priori formulation, testing and refinement of different hypotheses about the inverse relation between the surface ECG and various cardiac electrophysiological properties including: action potential duration, refractory period, upstroke velocity, amplitude, conduction velocity, and tissue conductivity. These electrophysiological parameters can be varied in many different ways, with related surface ECGs simultaneously computed in a very short time, thus elucidating the relation between the cardiac parameters and the measured surface potentials. The latter can furthermore be calculated as a standard 12-lead ECG system, signalaveraged ECG (SAECG), body surface potential mapping (BSPM) or any other lead system.

This methodology can be useful, for example, in the development of new diagnostic tools that could inversely assess cardiac properties from the associated surface ECG. Furthermore, our model can be used for the development and optimization of different signal processing tools such as QRST cancellation and atrial-ventricular source separation by isolating atrial from ventricular activity, as suggested in the modeling study of the Lausanne Heart Group [5].

Today, high dimensional 3D models prevail in cardiac mono and bidomain modeling, and with appropriate graphics rendering, these models appear more realistic and appealing than $1 \mathrm{D}$ and 2D models. $[1,3,6]$ While equivalent epicardial surface source models such as ECGSIM [7] offer high computational speed, this is achieved at the cost of being unable to simulate active propagation of activation in the myocardium, modify the tissue conductivity, or simulate therapeutic interventions for cardiac arrhythmias such as defibrillation, as bidomain models can do. However, it is not always necessary to use high dimensional, all-inclusive, highly detailed cardiac models to gain insight into the cardiac function. Even so, the lowest possible dimension models should be developed first and progressively expanded to higher dimensional and more complex models [8-10].

Up until the present study, there has been no general purpose 2D electrophysiological model of the whole heart and torso developed [3], probably due to a fact that 2D models are considered overly simplified to generate realistic ECG morphologies. 2D cardiac electrophysiology models have mainly been used for tissue modeling $[1,11]$. Nonetheless, 2D models offer a good compromise between computational load and model complexity, and they should be considered as an important stepping-stone towards more anatomically-accurate and functionally-detailed models [8]. It is interesting to examine how simple a cardiac 2D electrophysiological model needs to be to reproduce realistic ECG morphologies. 


\section{METHODS}

A simplified 2D computational model of the heart was developed, including regions defining the torso, lungs and myocardium with atria, ventricles, septum, and heart chamber cavities (Fig.1.). All electrical conductivities are given in Table 1. and were obtained from the literature [12, 13]. Four ECG electrodes were placed at the vertices of the rectangular torso to simulate surface potentials at the position of the left arm (LA), the right arm (RA), the left leg (LL) and the right leg (RL) or ground (Fig.1.). The right leg electrode was set to be the ground reference electrode so the potential $V$ at that node was fixed to $0 \mathrm{~V}$.

Outside the myocardium, the governing equation for the extracellular voltage $V$ in the passive volume conductor domains was given by

$$
\nabla \cdot\left(-\sigma_{b} \nabla V\right)=0
$$

where $\sigma_{b}$ is the relevant electrical conductivity within the torso, lungs and the cardiac chambers, respectively, as given in Table 1., and does not apply within the walls of the heart itself. All external edges of the rectangular domain (i.e., the torso) were electrically insulated. All the edges in contact with the heart were set to $V=V_{e}$ where $V_{e}$ is the extracellular voltage within the heart.

Three Einthoven leads $V_{I}, V_{I I}$ and $V_{I I I}$ from the standard 12-lead system were calculated as: $V_{I}=V_{L A}-V_{R A}$, $V_{I I}=V_{L F}-V_{R A}, V_{I I I}=V_{L F}-V_{L A}$ but other lead systems can be obtained, if desired, by placing additional electrodes on the torso, e.g., for the precordial leads or by implementing additional calculations, e.g., the augmented leads. Nevertheless, the electrical activity and the projections of the heart lead vector are limited to the frontal plane due to topology of the 2D model.

Table 1 . Conductivity values for various model tissues.

\begin{tabular}{llll}
\hline \hline Subdomain & $\begin{array}{l}\text { Conductivity } \\
{[\mathrm{S} / \mathrm{m}]} \\
{[12]}\end{array}$ & $\begin{array}{l}\text { Conductivity } \\
{[\mathrm{S} / \mathrm{m}]} \\
{[13]}\end{array}$ & $\begin{array}{l}\text { Conductivity } \\
{[\mathrm{S} / \mathrm{m}]} \\
\text { Our cardiac } \\
\text { model. }\end{array}$ \\
\hline Heart & 0.054 & $\begin{array}{l}0.400 \\
\text { (longitudinal) }\end{array}$ & 0.05 \\
& & 0.179 (transversal) & \\
Blood & 0.7 & 0.625 & 0.7 \\
Lungs & 0.203 (deflated) & 0.050 & 0.04 \\
& 0.039 (inflated) & 0.526 (parallel) & N/A \\
Muscle & 0.202 & 0.076 (normal) & \\
& & 0.040 & $\mathrm{~N} / \mathrm{A}$ \\
Fat & 0.012 & $\mathrm{~N} / \mathrm{A}$ \\
Bone & 0.000975 (marrow) & 0.006 & 0.2 \\
Torso & $\mathrm{N} / \mathrm{A}$ & $\mathrm{N} / \mathrm{A}$ & \\
\hline \hline
\end{tabular}

We used the modified FitzHugh-Nagumo (FHN) equations to simulate the various myocyte electrical activities. In comparison with other more accurate cardiac cell models [14] specialized often for only a particular heart region (with more than 10 variables at least) we used the same FHN equation (3 variables only) for all heart regions, allowing us computational simplicity and controllability, since they allowed the ready control of cardiac cell electrophysiological properties such as action potential duration and refractory period with only simple parameter modifications.

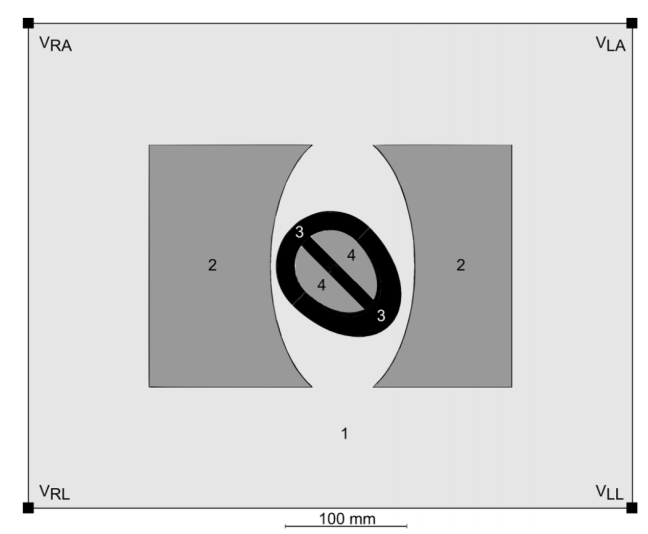

Fig.1. 2D geometry of the model comprising: $1=$ torso, $2=$ lungs, $3=$ atria and ventricles with $4=$ cardiac chambers, observed in the frontal plane. The ECG electrodes are placed at the corners of the torso indicating a potential $(\mathrm{V})$ of: the right $\operatorname{arm}\left(\mathrm{V}_{\mathrm{RA}}\right)$, the left arm $\left(\mathrm{V}_{\mathrm{LA}}\right)$, the left leg $\left(\mathrm{V}_{\mathrm{LL}}\right)$ and the right leg $\left(\mathrm{V}_{\mathrm{RL}}\right)$ or ground.

Within the myocardial tissues, the bidomain model was defined by three dependent variables: $V_{e}-$ the extracellular potential, $V_{i}-$ the intracellular potential, and $u-$ an excitation variable, governing cellular activation in the cardiac walls, including the SAN. The bidomain equations employed utilize FHN-type equations for cellular activation $[15,16]$ with slight modifications. For each region of the heart they are given by:

$$
\begin{aligned}
& \frac{\partial V_{e}}{\partial t}-\frac{\partial V_{i}}{\partial t}+\nabla\left(-\sigma_{e} \nabla V_{e}\right)=i_{i o n} \\
& \frac{\partial V_{i}}{\partial t}-\frac{\partial V_{e}}{\partial t}+\nabla\left(-\sigma_{i} \nabla V_{i}\right)=-i_{i o n} \\
& \frac{\partial u}{\partial t}=k e\left[\frac{\left(V_{m}-B\right)}{A}-d u-b\right]
\end{aligned}
$$

where $V_{m}=V_{i}-V_{e}$, with $\sigma_{i}, \sigma_{e}$ denote the extracellular and intracellular bidomain conductivities, respectively, $a, b, c_{1}, c_{2} d, e, k, A, B$ are parameters, and $i_{i o n}$ is the cell membrane ionic current, defined according to:

$$
i_{\text {ion }}=k c_{1}\left(V_{m}-B\right)\left[a-\frac{\left(V_{m}-B\right)}{A}\right]\left[1-\frac{\left(V_{m}-B\right)}{A}\right]+k c_{2} u
$$

within the SAN and

$$
i_{\text {ion }}=k c_{1}\left(V_{m}-B\right)\left[a-\frac{\left(V_{m}-B\right)}{A}\right]\left[1-\frac{\left(V_{m}-B\right)}{A}\right]+k c_{2} u\left(V_{m}-B\right)(6)
$$

within the walls of the atria, ventricles, atrioventricular node (AVN), His bundle, bundle branches and Purkinje fibers. 


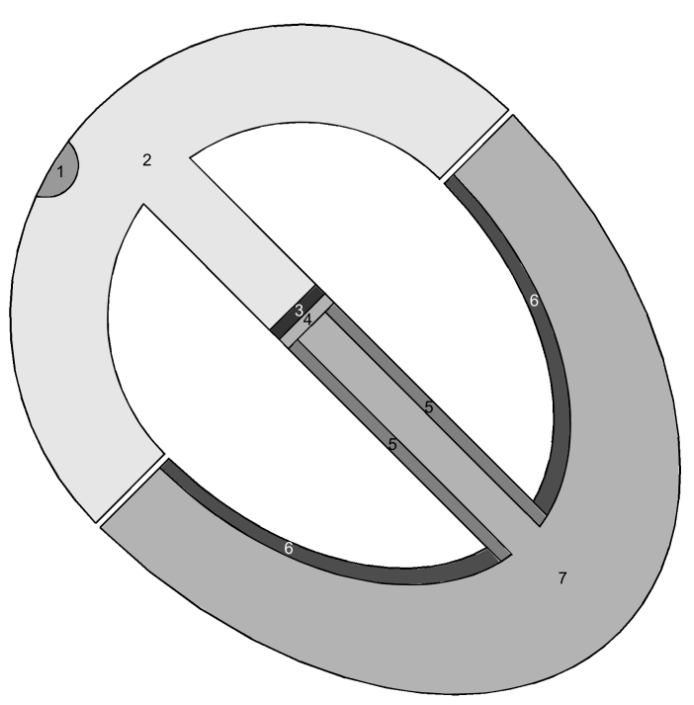

Fig.2. Subdomains of the heart: $1=$ sinoatrial node, $2=$ atria, $3=$ atrioventricular node, $4=$ His bundle, $5=$ bundle branches, $6=$ Purkinje fibers, $7=$ ventricles.

Table 2. Model parameters. SAN, avn, , bnl, pkj refer to the sinoatrial node, atrioventricular node, His bundle, bundle branches, and Purkinje fibers, respectively.

\begin{tabular}{|c|c|c|c|c|c|c|c|}
\hline $\begin{array}{l}\text { Para- } \\
\text { meters }\end{array}$ & SAN & Atria & AVN & HIS & $\mathrm{BNL}$ & PKJ & $\begin{array}{l}\text { Ventri- } \\
\text { cles }\end{array}$ \\
\hline $\mathrm{a}$ & -1 & 0.13 & 0.13 & 0.13 & 0.13 & 0.13 & 0.13 \\
\hline b & $-0.29 \mathrm{e}-3$ & 0 & 0 & 0 & 0 & 0 & 0 \\
\hline$c_{1}$ & 1.9 & 2.6 & 2.6 & 2.6 & 2.6 & 2.6 & 2.6 \\
\hline$c_{2}$ & $1 \mathrm{e}-3$ & 1 & 1 & 1 & 1 & 1 & 1 \\
\hline $\mathrm{d}$ & 0 & 1 & 1 & 1 & 1 & 1 & 1 \\
\hline e & $\begin{array}{l}0.060 \\
\text { for } \\
V_{\mathrm{m}^{\prime}}>=0 \\
0.300 \\
\text { for } \\
V_{\mathrm{m}^{\prime}}<0\end{array}$ & 0.010 & 0.010 & 0.0045 & 0.0028 & 0.0043 & 0.0050 \\
\hline $\mathrm{A}[\mathrm{mV}]$ & 35 & 140 & 140 & 140 & 140 & 140 & 140 \\
\hline $\mathrm{B}[\mathrm{mV}]$ & -30 & -85 & -85 & -85 & -85 & -85 & -85 \\
\hline $\mathrm{k}$ & 1000 & 1000 & 1000 & 1000 & 1000 & 1000 & 1000 \\
\hline$\sigma_{\mathrm{e}}\left[\mathrm{mS} \cdot \mathrm{m}^{-1}\right]$ & 0.5 & 8 & 0.5 & 10 & 15 & 35 & 8 \\
\hline$\sigma_{\mathrm{i}}\left[\mathrm{mS} \cdot \mathrm{m}^{-1}\right]$ & 0.5 & 8 & 0.5 & 10 & 15 & 35 & 8 \\
\hline
\end{tabular}

Parameters of the model have domain-specific values in different regions of the heart, and are listed in Table 2. The model parameters for the FHN type cardiac cells are taken from [15] while action potential duration and propagation delays due to different conductivities in various tissue types were derived from the data available in [13]. Parameter $e$ mainly regulates the action potential duration, whilst conductivity parameters $\sigma_{e}, \sigma_{i}$ mainly control the conduction velocity of different specialized cardiac tissue. For example, the lower conductivity in the AVN induces an appropriate delay in impulse conduction to the ventricles.

Initial values of all model variables are given in TABLE 3 . The SAN region is more depolarized than other regions of the heart, therefore, SAN cells are the first to reach the activation threshold.
Table 3. Model Initial values. SAN, avn, his, bnl, pkj refer to the sinoatrial node, atrioventricular node, His bundle, bundle branches, and Purkinje fibers, respectively.

\begin{tabular}{llllllll}
\hline \hline $\begin{array}{c}\text { Para- } \\
\text { meters }\end{array}$ & SAN & Atria & AVN & HIS & BNL & PKJ & $\begin{array}{c}\text { Ventri- } \\
\text { cles }\end{array}$ \\
\hline $\mathrm{V}_{\mathrm{i}}[\mathrm{V}]$ & -0.06 & -0.085 & -0.085 & -0.085 & -0.085 & -0.085 & -0.085 \\
$\mathrm{~V}_{\mathrm{e}}[\mathrm{V}]$ & 0 & 0 & 0 & 0 & 0 & 0 & 0 \\
$\mathrm{u}$ & 0 & 0 & 0 & 0 & 0 & 0 & 0 \\
\hline \hline
\end{tabular}

The boundary conditions on all edges in contact with the torso and cardiac chambers are zero-flux for $V_{i}$, therefore, $\boldsymbol{n} \cdot \boldsymbol{\Gamma}=0$ where $\boldsymbol{n}$ is the unit outward normal vector on the boundary and $\boldsymbol{\Gamma}$ is the flux vector through that boundary, equal to $\boldsymbol{\Gamma}=-\sigma_{i} \cdot \partial V_{i} / \partial \boldsymbol{n}$, where $\sigma_{i}$ is the intracellular conductivity. For the variable $V_{e}$ the inward flux on these edges is equal to the outward current density $\boldsymbol{J}$ from the torso conduction volume, therefore, $-\sigma_{e} \cdot \partial V_{e} / \partial \boldsymbol{n}=\boldsymbol{n} \cdot \boldsymbol{J}$, where $\sigma_{e}$ is the extracellular conductivity in the myocardium.

Our simplified 2D cardiac model was implemented using COMSOL Multiphysics 4.2a finite element software (COMSOL AB, Sweden). The 2D cardiac model consisted of a finite-element mesh with 8174 quadratic order elements having 28403 degrees of freedom. The time dependent solver took about 6 minutes to resolve 1 simulated second with $1 \mathrm{~ms}$ resolution of the ECG and the transmembrane potentials. The accuracy and stability of the model were tested for finer mesh resolutions, and it was found that for finer spatial and temporal discretizations the model ECG converged to the same solution (results not shown). The simulations were performed on an Intel Core i7-970 processor workstation with $2 \times 6$ cores at about 100 Gflops. Shared memory parallelism on a multicore processor can further improve computation performance, especially during the parameter sweeping study, although not in the proportion to the number of cores used [17].

To validate the model, we varied a range of intrinsic myocardial electrophysiological properties to examine whether these variations reproduced known effects on ECG morphology. These were: (a) a lowering ventricular tissue conductivity (and consequently ventricular conduction velocity) to ascertain the effect on ECG morphology, (b) prolongation of QT interval due to delayed ventricular repolarization, and (c) P wave shortening induced by early atrial repolarization and consequent shortening of atrial action potential duration.

\section{RESULTS}

Rhythmic activation occurs spontaneously in the 2D cardiac model, originating in the pacemaker region of the SAN with the electrical impulse spreading throughout the atria before reaching the AVN where the excitation wave front is delayed until the whole atria is activated. Subsequently the AVN activates the His bundle from where the activation spreads to the bundle branches, the Purkinje fibers, and the whole ventricles (Fig.3.). 


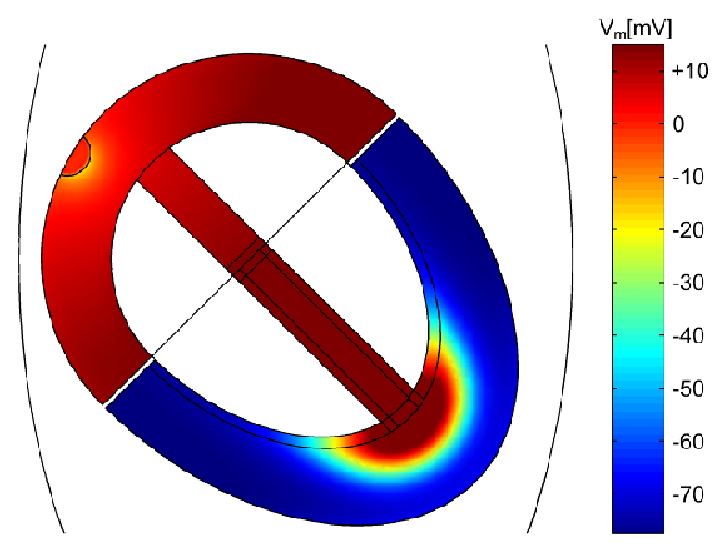

Fig.3. Transmembrane potential $\left(\mathrm{V}_{\mathrm{m}}\right)$ in the heart at the moment when the depolarization excitation wave front has just spread into the left and right ventricles, and when atrial repolarization is about to begin. The $\mathrm{V}_{\mathrm{m}}$ ranges between $-85 \mathrm{mV}$ and $20 \mathrm{mV}$ as indicated by the colorbar.
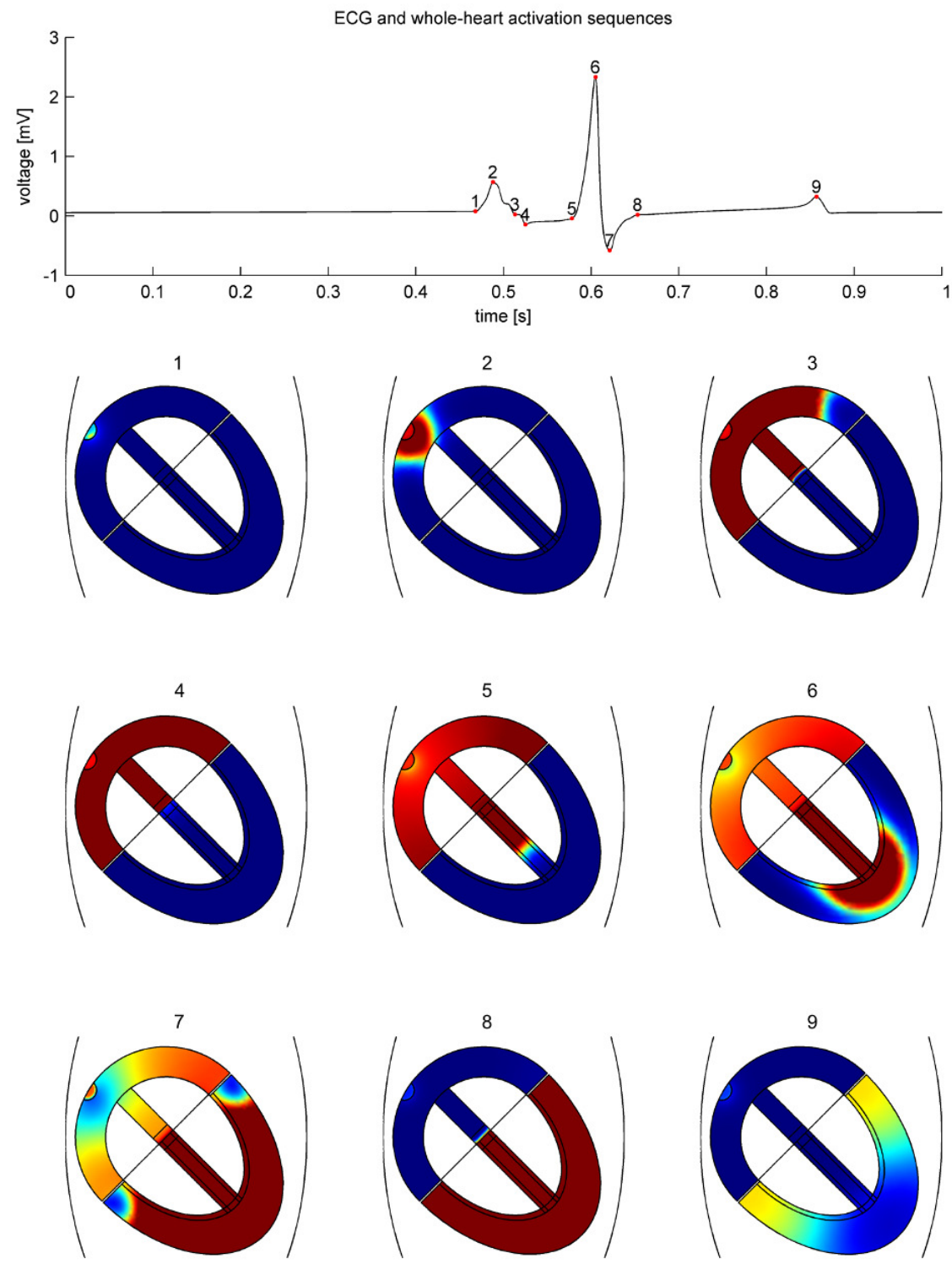

Fig.4. Simulated lead II ECG signal and simultaneous whole-heart activation sequences. Numbers and markers on the ECG indicate the time epochs in which corresponding activation sequences below are illustrated. 


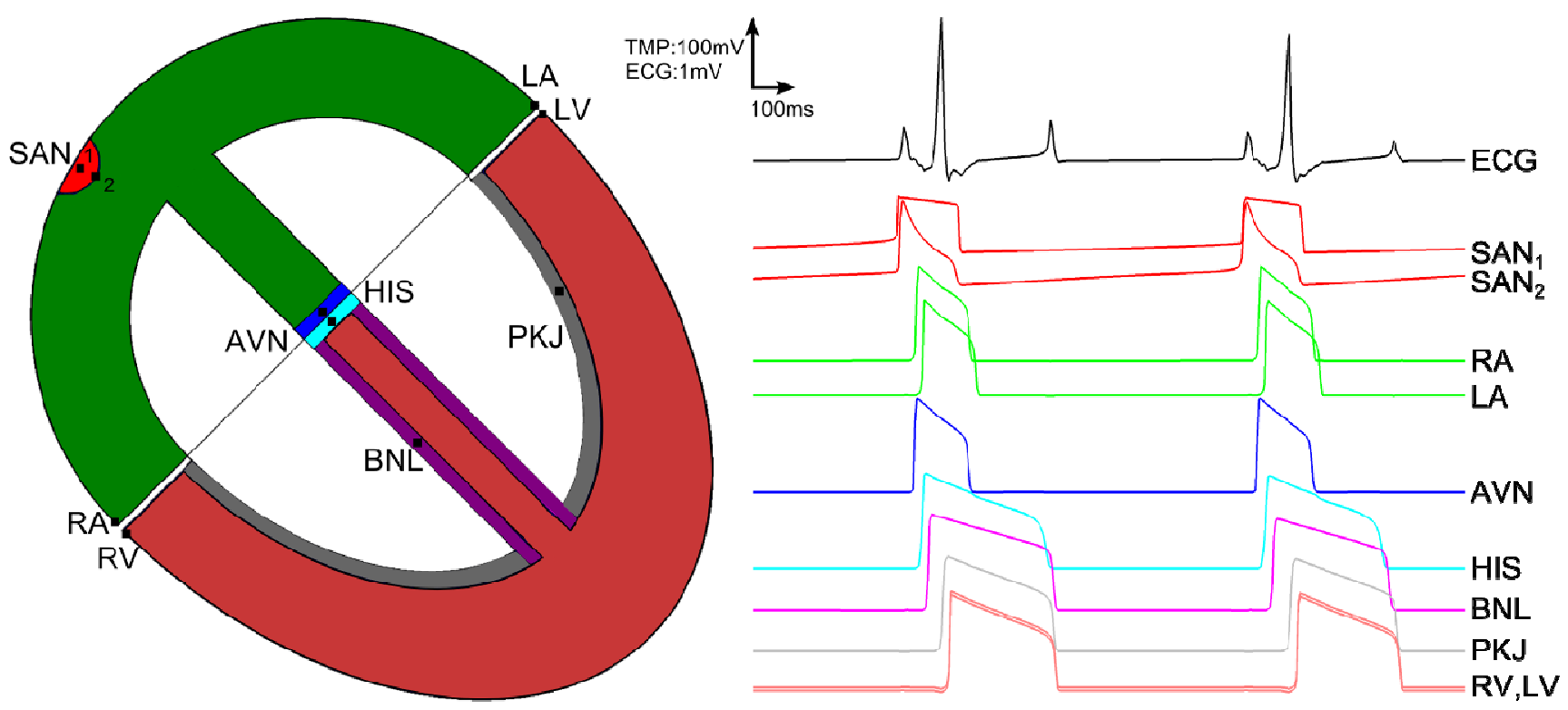

Fig.5. Simulated ECG and transmembrane potential (TMP) waveforms of the 2D cardiac model. Left figure illustrates probe locations (black dots) at various sites in the heart: the sino-atrial node (SAN), the right atrium (RA), the left atrium (LA), the atrioventricular node (AVN), the His bundle (HIS), the bundle branches (BNL), the Purkinje fibers (PKJ), the right ventricle (RV) and the left ventricle (LV). The right panels illustrate the simulated lead II ECG waveform and transmembrane action potentials at the various probe locations.

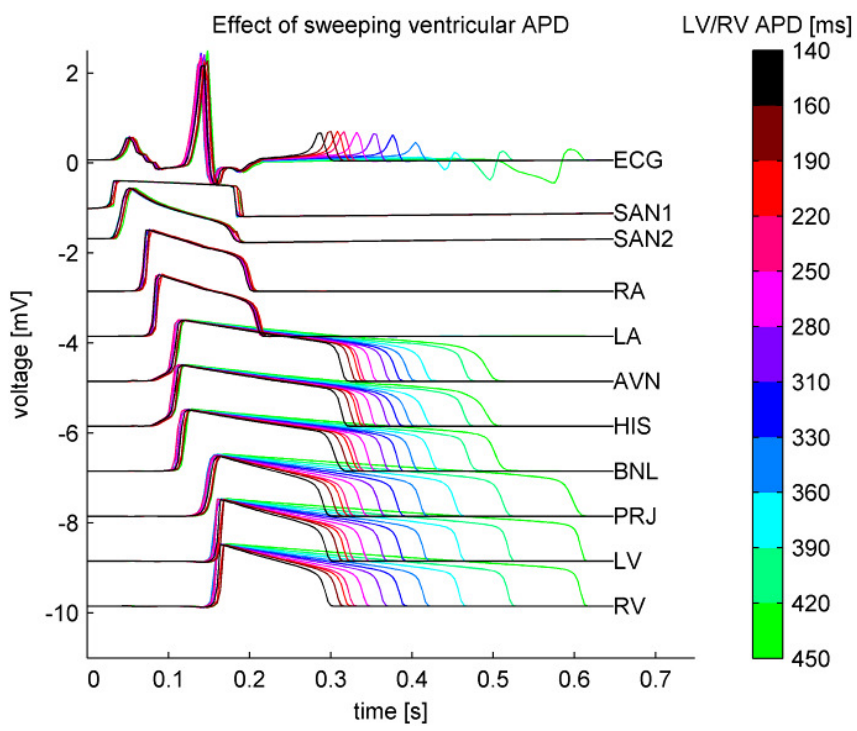

Fig.6. Effect of sweeping ventricular action potential duration (APD). The colorbar indicates the levels of APD in the RV/LV and the corresponding colors for plotting the ECG and TMPs/100 at those levels.

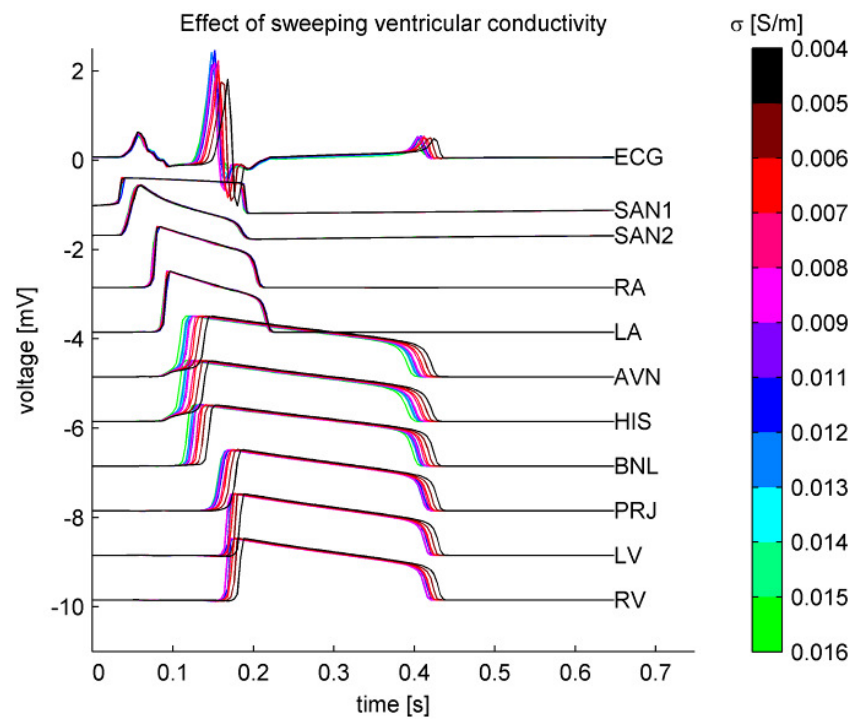

Fig.7. Effect of sweeping ventricular conductivity. All other parameters are set to their default values given in Table 2. The colorbar indicates the levels of the conductivity parameter and the corresponding colors used for plotting the ECG and TMPs/100 at those levels. 


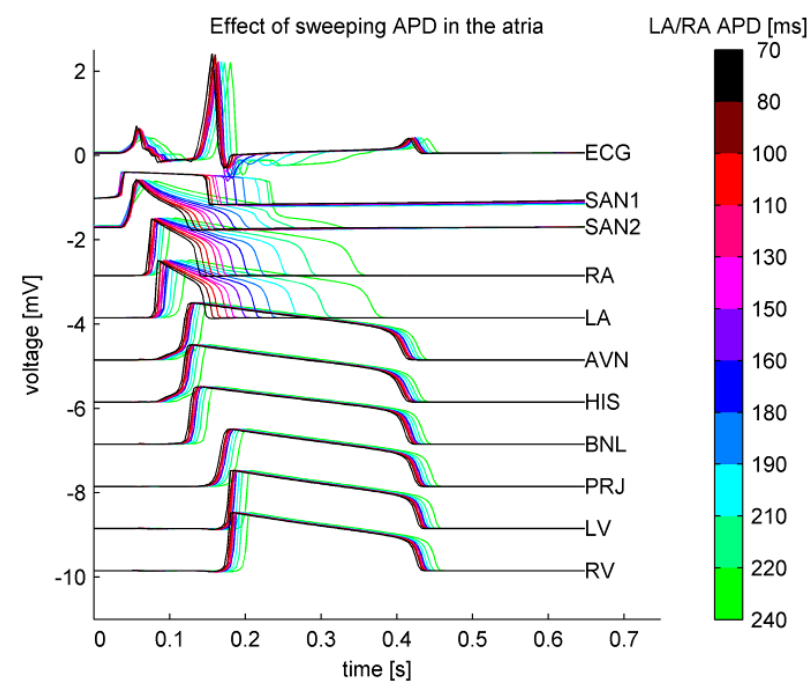

Fig.8. Sweeping action potential duration in the atria only. The colorbar indicates levels of the swept parameter and the corresponding colors used for plotting the ECG and TMPs/100 at those levels.

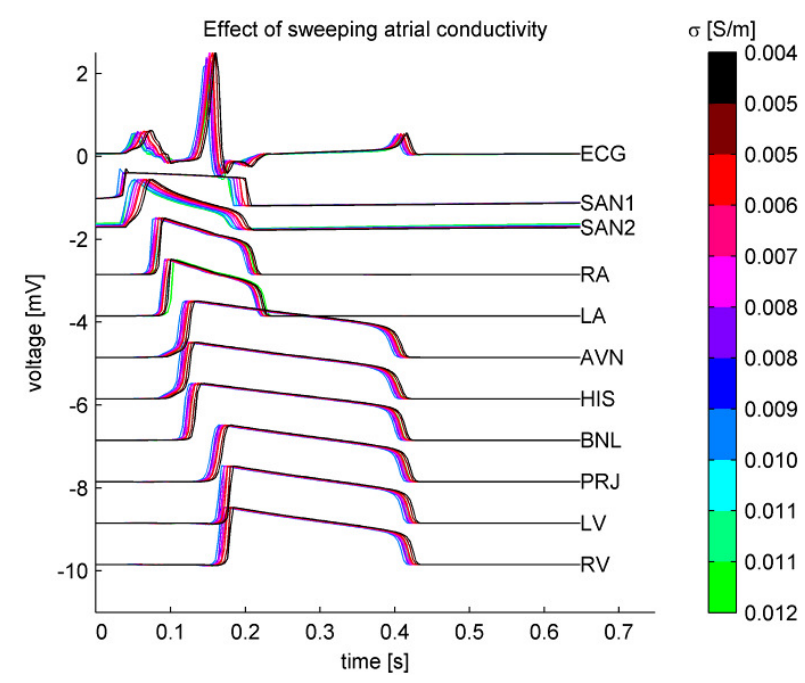

Fig.9. Sweeping atrial tissue conductivity. All other parameters are set to their default values given in Table 2. The colorbar indicates levels of the swept parameter and the corresponding colors used for plotting the ECG and TMPs/100 at those levels.

Fig.. illustrates important characteristic time epochs in the simulated lead II ECG and the corresponding state of overall heart activation at those times. The activation sequences characterize the repolarization and depolarization wavefronts of the propagating transmembrane potential. It can be observed in epoch 3 that the AVN imposes a time delay until the whole atria are activated, while in epoch 7 we can observe the overlap between the ongoing ventricular depolarization coinciding with the beginning of atrial repolarization.

Fig.5. illustrates the computed lead II electrocardiogram (ECG) and the corresponding transmembrane action potentials (TMPs) acquired at various locations in the heart over a simulated interval of 1.6 seconds. The excitation originating in the SAN is spontaneous and periodic, while the ECG waveform remains stable and morphologically realistic.

Fig.6. illustrates results of sweeping action potential duration (APD) in the ventricles by linearly varying parameter $e$ from Table 2 . in ten steps within the range from 0.005 to 0.02 . As expected, the results indicate that shortening the APD in all regions of the ventricles produces a shortening of the QT interval on the lead II ECG.

Fig.7. illustrates the effect of varying ventricular conductivity on the ECG waveform. The conductivity is directly related to the conduction velocity. As expected, lowering the conductivity in the ventricles produces delayed action potentials and consequently delayed ventricular depolarization and repolarization.

Fig.8. illustrates the effect of changing the APD only in the atria by linearly varying parameter $e$ from Table 2 . in ten steps within the range from 0.005 to 0.02 . Besides its effect on APD shortening, parameter $e$ affects the AP plateau slope as well, which becomes more triangular-like. Shorter APDs and action potential waveshapes with no plateau are often associated with patients prone to atrial fibrillation $[18,19]$.

Fig.9. illustrates the effect of sweeping atrial tissue conductivity, which consequently sweeps atrial conduction velocity. As can be seen, a decrease in atrial conductivity leads to a delay in the onset of the $\mathrm{P}$ wave as well as prolonging the $\mathrm{P}$ wave duration. Along with APD shortening, decreased myocardial conductivity can lead to reentrant activation underlying atrial fibrillation $[5,19]$.

\section{DISCUSSION}

The results of this study indicate that the simplified 2D bidomain model of the heart can generate realistic ECG morphologies, offering a good compromise between computational efficiency, complexity and accuracy (Fig.5.). ECG simulations performed by sweeping APD and conductivity in different heart regions are consistent with expectations, and indicate that the proposed model can be used as a tool in basic research and education. An ideal computational model has to be as simple as possible and as complex as necessary for the particular investigation at hand [8]. Therefore, to validate the model we have investigated the relation between $\mathrm{APD}$, tissue conductivity and the resulting ECG.

For example, it is well known in clinical practice that the QT interval is a function of APD and that these two features are very closely correlated [20]. This relationship was simulated using the 2D model of this study, in order to validate the model and confirm that APD prolongation in all regions of ventricles leads to a longer QT interval.

The same numerical experiment with sweeping APD duration was repeated in the atria. In an earlier study [21, 22], we claimed that changes in the PQ segment level (defined as elevation / depression of the segment level between $\mathrm{P}$ wave end and $\mathrm{Q}$ wave onset) and the falling $\mathrm{P}$ wave slope are related to premature atrial repolarization, APD shortening and loss of AP plateau, and this is often found in patients prone to atrial fibrillation $[21,22]$. These 
hypotheses were tested again with the $2 \mathrm{D}$ cardiac model of this study and the results (Fig.8.) suggest that the premature atrial repolarization, APD shortening and loss of AP plateau indeed have an effect on the falling edge of the $\mathrm{P}$ wave, the PQ segment level and the $\mathrm{P}$ wave duration. This is an interesting finding, since a decrease in atrial conductivity, with the consequent decrease in atrial conduction velocity (Fig.8.) suggests a prolongation in the $\mathrm{P}$ wave duration. Since patients prone to atrial fibrillation exhibit both phenomena: shortening of APD and decrease in conduction velocity [19], these two lead to opposite effects. Therefore, the results of this study suggest that the prediction of atrial fibrillation (AF) based on one feature only, such as the $\mathrm{P}$ wave duration information, may be incomplete. Therefore, to improve the sensitivity and specificity of AF prediction, we suggest that AF prediction and classification based on $\mathrm{P}$ wave duration feature alone should be expanded and combined with an additional, independent feature such as, for example, the PQ segment level or the falling slope of the $\mathrm{P}$ wave, in order to add some new, independent information.

\section{CONCLUSIONS}

We have developed a simplified and computationally efficient 2D cardiac model of whole heart electrical activity with spontaneous sinoatrial node pacemaking activity and realistic ECG morphologies. Our 2D model offers a good compromise between computational load and model complexity, and is suitable to be used for both education and research such as, for example, for rapid hypothesis testing and model development as an intermediate step towards development of more anatomically detailed 3D models. The bidomain model has the advantage of being able to directly simulate the active spread of activation in the heart, and can be used to investigate the coupling between tissue electrical properties and the whole-heart ECG observed on the torso. It can also be used to simulate the efficacy of various therapeutic interventions such as electrical defibrillation or the action of ion channel drugs on cardiac arrhythmias. Furthermore, such a model could also be used to evaluate the pro-arrhythmic susceptibility of individuals from changes in their ECG morphology. Such simulations are not possible using simple epicardial source models of ECG generation such as ECGSIM.

Future improvements to the model will include introduction of an appropriate random spatial variation of ventricular APD to improve T wave shape and extending the simplified geometry to $3 \mathrm{D}$, in order to reproduce a more complete range of reentrant dynamics. A 3D model could also provide for a more realistic topology of current flow around the lungs, although this did not appear to be a significant factor in reproducing realistic ECG morphologies, as evidenced by the reasonable ECG waveforms obtained from the 2D model of this study. Simplified bidomain models of ECG generation will provide important tools for estimating pro-arrhythmic susceptibility from changes in the ECG morphology.

Although the model was able to reproduce reasonable
ECG morphologies, the shape of the $\mathrm{T}$ wave tended to be narrower than that commonly observed. This property of the model was likely due to the nature of the uniform AP implemented throughout the ventricles, such that repolarization occurred nearly simultaneously at all points throughout the ventricular myocardium. The $\mathrm{T}$ wave duration could be widened by introducing some randomness in ventricular AP parameters (such as parameter $e$ ) which would lead to heterogeneous AP durations and a spread in ventricular repolarization times. Such improvements, if desired, could readily be introduced in future versions of the model.

\section{ACKNOWLEDGMENT}

This study was supported by the Australian Department of Education, Employment and Workplace Relations (DEEWR) and the Ministry of Science, Education and Sport (MZOS) of the Republic of Croatia, under grant \#0360362979-1554. During the academic visit, SS was holder of the Australian Government's Endeavour Award fellowship (\#ERF_PDR 3203_2012) for the postdoctoral research at the University of New South Wales (UNSW), Graduate School of Biomedical Engineering (GSBmE).

\section{REFERENCES}

[1] Pullan, A.J., Buist, M.L., Cheng, L.K. (2005). Mathematically Modelling the Electrical Activity of the Heart - From Cell to Body Surface and Back Again. World Scientific.

[2] Seemann, G. et al. (2010). Electrophysiological modeling for cardiology: Methods and potential applications. it - Information Technology, 52 (5), 242249.

[3] Trayanova, N.A. (2011). Whole-heart modeling Applications to cardiac electrophysiology and electromechanics. Circulation Research, 108, 113128.

[4] Trudel, M.-C. et al. (2004). Simulation of QRST integral maps with a membrane-based computer heart model employing parallel processing. IEEE Transactions of Biomedical Engineering, 51 (8), 1319-1329.

[5] Jacquemet, V., van Oosterom, A., Vesin, J., Kappenberger, L. (2006). Analysis of electrocardiograms during atrial fibrillation. IEEE Engineering in Medicine and Biology Magazine, 25 (6), 79-88.

[6] Pfeifer, B. et al. (2007). A training whole-heart model for simulating propagation and ECG patters. Biomedical Signal Processing and Control, 2 (4), 323330.

[7] Van Oosterom, A., Oostendorp, T.F. (2003). ECGSIM: An interactive tool for studying the genesis of QRST waveforms. Heart, 90 (2), 165-168.

[8] Garny, A., Noble, D., Kohl, P. (2005). Dimensionality in cardiac modelling. Progress in Biophysics and Molecular Biology, 87 (1), 47-66. 
[9] Sovilj, S., Magjarevic, R., Lovell, N., Dokos, S. (2013). Realistic 3D bidomain model of whole heart electrical activity and ECG generation. In Computing in Cardiology Conference, 22-25 September 2013. IEEE, 377-380.

[10] Sovilj, S., Magjarevic, R., Lovell, N., Dokos, S. (2013). A simplified 3D model of whole heart electrical activity and 12-lead ECG generation. Computational and Mathematical Methods in Medicine, article ID 134208-10.

[11] Gibbons Kroeker, C.A., Adeeb, S., Tyberg, J.V., Shrive, N.G. (2006). A 2D FE model of the heart demonstrates the role of the pericardium in ventricular deformation. American Journal of Physiology - Heart and Circulatory Physiology, 291 (5), H2229-2236.

[12] Gabriel, S., Lau, R.W., Gabriel, C. (1996). The dielectric properties of biological tissues: II. Measurements in the frequency range $10 \mathrm{~Hz}$ to 20 GHz. Physics in Medicine and Biology, 41, 22512269.

[13] Malmivuo, J., Plonsey, R. (1995). Bioelectromagnetism: Principles and Applications of Bioelectric and Biomagnetic Fields. Oxford University Press.

[14] Fenton, F.H., Cherry, E.M. (2008). Models of cardiac cell. Scholarpedia, 3 (8), 1868.

[15] Dokos, S., Cloherty, S.L., Lovell, N.H. (2007). Computational model of atrial electrical activation and propagation. In Annual International Conference of the IEEE Engineering in Medicine and Biology Society. IEEE, 908-911.
[16] Rogers, J.M., McCulloch, A.D. (1994). A collocationGalerkin finite element model of cardiac action potential propagation. IEEE Transactions on Biomedical Engineering, 41, 743-757.

[17] Petra, N., Gobbert, K.M. (2009). Parallel performance studies for COMSOL multiphysics using scripting and batch processing. In Proceedings of the COMSOL Conference 2009 Boston.

[18] Janse, M.J. (1997). Why does atrial fibrillation occur? European Heart Journal, 18, C12-C18.

[19] Nattel, S. (2002). New ideas about atrial fibrillation 50 years on. Nature, 415, 219-226.

[20] Seed, W.A. et al. (1987). Relation of human cardiac action potential duration to the interval between beats: Implications for the validity of rate corrected QT interval (QTc). British Heart Journal, 57, 32-37.

[21] Sovilj, S., Rajsman, G., Magjarevic, R. (2011). ECG based prediction of atrial fibrillation using support vector classifier. Automatika - Journal for Control, Measurement, Electronics, Computing and Communications, 52 (1), 58-67.

[22] Sovilj, S., Van Oosterom, A., Rajsman, G., Magjarevic, R. (2010). ECG based prediction of atrial fibrillation development following coronary artery bypass grafting. Physiological Measurement, 31, 663677.

Received November 11, 2013. Accepted June 28, 2014. 